\title{
THE ANALYSIS OF OBJECT-BASED CHANGE DETECTION IN MINING AREA: A CASE STUDY WITH PINGSHUO COAL MINE
}

\author{
Miaolin Zhang ${ }^{\mathrm{a}}$, Wei Zhou, ${ }^{\mathrm{a},}$, Yifan Li ${ }^{\mathrm{b}}$

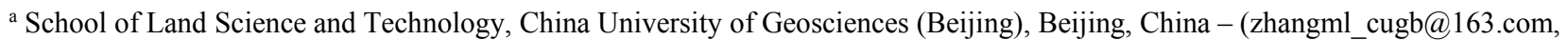 \\ zhouw@cugb.edu.cn) \\ b School of Environment, Beijing Normal University, Beijing, China - liyf_simlab@163.com
}

Commission III, WG III/6

KEY WORDS: Land use/cover monitoring, Object-based classification, Multi-temporal/multi-date object change detection, Openpit coal mine, Land reclamation, Remote sensing

\begin{abstract}
:
Accurate information on mining land use and land cover change are crucial for monitoring and environmental change studies. In this paper, RapidEye Remote Sensing Image (Map 2012) and SPOT7 Remote Sensing Image (Map 2015) in Pingshuo Mining Area are selected to monitor changes combined with object-based classification and change vector analysis method, we also used $\mathrm{R}$ in highresolution remote sensing image for mining land classification, and found the feasibility and the flexibility of open source software. The results show that (1) the classification of reclaimed mining land has higher precision, the overall accuracy and kappa coefficient of the classification of the change region map were $86.67 \%$ and $89.44 \%$. It's obvious that object-based classification and change vector analysis which has a great significance to improve the monitoring accuracy can be used to monitor mining land, especially reclaiming mining land; (2) the vegetation area changed from $46 \%$ to $40 \%$ accounted for the proportion of the total area from 2012 to 2015 , and most of them were transformed into the arable land. The sum of arable land and vegetation area increased from $51 \%$ to $70 \%$; meanwhile, build-up land has a certain degree of increase, part of the water area was transformed into arable land, but the extent of the two changes is not obvious. The result illustrated the transformation of reclaimed mining area, at the same time, there is still some land convert to mining land, and it shows the mine is still operating, mining land use and land cover are the dynamic procedure.
\end{abstract}

\section{INTRODUCTION}

Mining area caused a great deal of changes in landscape structure and enormous environmental disturbances, among them open-pit coal mine is one of the greatest landscapealtering activities, and it's difficult to restore surface coal mine to the original ecological landscape (Hendrychová and Kabrna 2016). There is no doubt that the coal mining expansion benefits the local GDP significantly, but also causes environmental degradation and destroy original ecosystem balance due to the destruction of original land cover types(Li, Yan et al. 2015). Scientific monitoring of coal mining areas, especially opencast coal mines, is of great theoretical and practical value for the new normal of China (Zhou et al. 2012)

To solve the conflict between the mining activities and the environmental protection in the region, after extracting the raw material, most countries claim mining companies to take reclamation projects for integrating landscape, and the surrounding areas. The impact of mining on the landscape is so significant that the reclamation of mining area should have different functions: production (forests, agricultural land), social (residential area, new constructions) and area of mining industry (Demirel, Emil et al. 2011). The traditional method for mine area monitoring which is to take the dynamic monitoring for land resource, requires a lot of manpower and material resources to conduct field investigation of mining areas. And it is no longer applicable to real-time monitoring and data updates for large-scale open-pit mine. Compared with the traditional method, the use of remote sensing in the mining area, especially reclamation land, can build a complete database to facilitate data collation, reduce costs and improve efficiency. Therefore change information is important for practical uses especially in mining area, including damage assessment, disasters monitoring, revegetation, reclamation, and land management.

Change detection is considered as monitoring the alterations of land cover and land use (Singh 1989). The processes of change detection are using multi-temporal remote sensing images to analyze differences in the state of features by observing them and then to quantify the changes. The remote sensing data is one of the major sources for change detection for acquiring data with sufficient area coverage, temporal frequency, digital format easy to compute and much more spatial and spectral resolutions to select (Hussain, Chen et al. 2013). There are many methods in change detection which are mainly pixel-based and object-based, and all of them are based on changes in the object of interest will alter the reflectance value or local texture. Since the 1970s, change detection has been applied abroad in mining area by using pixel-based classification in low-resolution remote sensing data (Boerner, DeMers et al. 1996). In China, these study began from the 1980s, Hu et al. (2005) analyzed the land use change in mining area using pixel-based method in Landsat TM and ETM data of Tangshan

If detailed change direction is required for a study, postclassification will be needed, Many pixel-based change detection methods, which have been used traditionally in large or medium scale, often using medium spatial resolution image such as Landsat Thematic Mapper (TM) are not considered appropriate for high-resolution remote sensing data, in which object-based analysis may be used more frequently. Highresolution remote sensing data (e.g. QuickBird, IKONOS, RapidEye) is used for local scale studies as it provides greater spatial resolution. The pixel-based techniques face a challenge on these High-resolution data (Lu, Moran et al. 2011, Chen, Hay et al. 2012). When undertaking a per-pixel classification a 
community scale, the spectral heterogeneity may lead to misclassified pixels appearing within classes creating a 'salt and pepper' effect (Myint, Gober et al. 2011). More recently, objectbased methods are more commonly used for change detection at the local scale such as mining area, for mining operations often occur at small spatial scales relative to other land cover changes (e.g. urban sprawl).

High spatial resolution remote sensing data allow us to obtain more detailed data in order to improve classification in the mining area. With suitable Remote sensing classification, we can get more accurate results of change detection. The pixelbased method only uses spectral information to classify, and cannot provide sufficient detail information in high spatial resolution remote sensing data for mining area. Unlike pixelbased classification, the object-based method first segments images into objects and then classifies objects by using spectral, textural features (Rodriguez-Galiano, Ghimire et al. 2012). Remote sensing change monitoring was used to monitor largescale areas by using low-resolution remote sensing images in the past, which has lower resolution, less information, and cannot conduct depth analysis of certain areas. So the objectbased backdating approach was put forward, which achieved higher accuracy for change monitoring.

Multi-temporal object-based technology has already been successfully applied in urban environment. Such as, damage monitoring is conducted by Al Khudhairy, etc. using pansharpened IKONOS data, while Chen and Hutchinson using panchromatic QuickBird imagery of urban buildings (Alkhudhairy, Caravaggi et al. 2005, Chen and Hutchinson 2007). The results of many researches have shown that objectbased classification can improve the classification accuracy so as to improve the accuracy of land use change detection results and provide more reliable technical support for mining area monitoring. So object-based classification and change detection can be applied in mining area monitoring.

Most procedures of remote sensing data can be handled readily in geographic information systems (GIS) or image processing software. Indeed, it is not important what exactly proprietary software is doing the procedures. $\mathrm{R}$ provides an alternative to some software for study and testing of remote sensing image processing algorithms. The flexibility of $\mathrm{R}$ makes it possible to apply, test and modify algorithms easily. The available graphical and statistical tools have already been convenient to anything available in common packages, making it straightforward to call algorithms. Tools for processing and display of spatial referenced data are widely available in $R(R$ Development Core Team 2011). Packages in $\mathrm{R}$ provide these capabilities for several commonly-used spatial formats.

In this paper, we use the data of RapidEye Remote Sensing Image and SPOT7 Remote Sensing Image to monitor the land use change in Pingshuo open-pit mine. Combined with objectbased classification and support vector machine in $\mathrm{R}$ to improve the efficiency of classification, to study high-resolution remote sensing data for mining area classification and the accuracy of change detection.

\section{MATERIAL}

\subsection{Study area}

Pingshuo open-pit mine $\left(112^{\circ} 10^{\prime} \mathrm{E} \sim 113^{\circ} 30^{\prime} \mathrm{E}\right.$ and $39^{\circ} 23^{\prime} \mathrm{N} \sim$ $\left.39^{\circ} 37^{\prime} \mathrm{N}\right)$ is located in the Shuozhou City of Shanxi Province, which covers an area of $360 \mathrm{~km}^{2}$ and its verified geological reserves are 12.75 billion tons in the latest prospecting. Designed to serving for 92 years (1985 2077), Pingshuo openpit mine has an annual output of the coal amounts to 65 million tons. The pit is sited in a fragile ecosystem, which is rather sensitive to environmental disturbance, and the plasticity of remaining self-stability is relatively small. The environment of open-cast mine is a fragile ecosystem, which is sensitive to and is difficult to maintain stability itself.

The study area (Figure. 1) in this paper with a total area of $30.43 \mathrm{~km}^{2}$, which includes the Antaibao west dump, Antaibao inner dump, Antaibao south dump, mining area and residential area. The mining area is sited in a fragile ecosystem in Loess Plateau, for the strongly eroded and less vegetation and has been reclaimed since the 1980s (Bai et al., 2008). And this mine has serious environmental problems (such as land collapse, soil erosion, soil desertification) caused by the lack of environmental protection.

In addition, Pingshuo open-pit mine is located in the northern part of Shanxi Province, which is one of the major sandstorm sources of Beijing and Tianjin. Therefore, a fragile ecosystem such as mining area will greatly endanger the local environment. It is obvious that the study area badly needs scientific monitoring and protection.

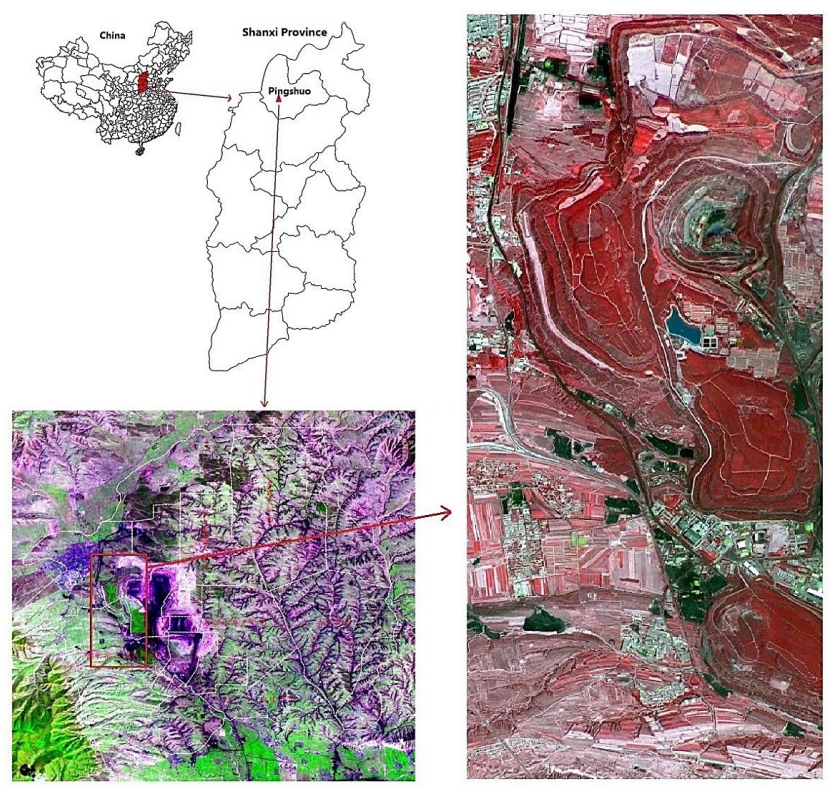

Figure 1. Study area: Pingshuo open-pit mine

\subsection{Data}

In this paper, we use RapidEye image $\left(t_{0}\right)$ and SPOT7 image $\left(t_{1}\right)$ as the based data. The former is collected in September 2012, which employed 5-band multispectral (blue, green, red, nearinfrared (NIR), red edge), and with a resolution of 4.27 meters. The latter is collected in August 2015, which provides both image products at 4 meters spatial resolution comprising multispectral bands of blue, green, red, and NIR and 1 meters panchromatic image. Both optical data sets are available as standard image products, i.e. radio metrically corrected, sensor corrected, and projected to the Earth's surface.

\begin{tabular}{|l|c|c|}
\hline & RapidEye & SPOT7 \\
\hline & $\mathrm{nm}$ & $\mathrm{nm}$ \\
Blue & $440-510$ & $455-525$ \\
Green & $520-590$ & $530-590$ \\
Red & $630-685$ & $625-695$ \\
NIR & $760-850$ & $760-890$ \\
Red Edge & $690-730$ & - \\
Pan & - & $455-745$ \\
\hline
\end{tabular}

Table 1. The band parameters of two sensors 
In addition, height information is available by means of a digital elevation model (DEM) in the study area, in order to reduce the effect of terrain on the classification accuracy. And the RapidEye and SPOT7 images are roughly consistent in the blue, green and NIR band so that their combination can be benefited to classification. Therefore this paper chooses the above bands of the RapidEye and SPOT7 images to build the data set in order to segment image objects.

\section{METHODOLOGY}

All process of this study is in Figure 2.

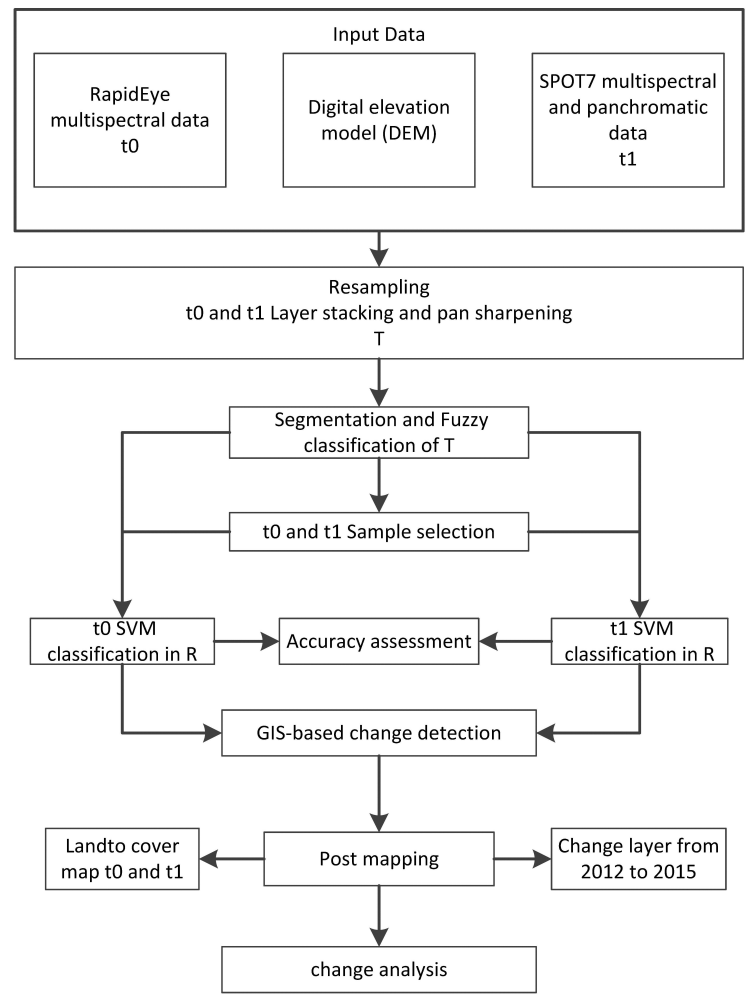

Figure 2. The process of classification and change analysis

\subsection{Preprocess}

Before classification and change detection, we need to preprocess the standard data products (both four-band multispectral and panchromatic) whose parameters is in Table 1 , and the processes include registration, resampling, radiometric, atmospheric correlation, and using DEM to reduce the effect of topography. Registration is a procedure that makes the two data from different sensors georeferenced and normalized. Resampling refers to take two images at the same resolution and have the same pixel number. Radiometric, atmospheric correlation, and using DEM to reduce the effect of topography are the procedures to improve images accuracy.

Panchromatic data were used to pan-sharpen the multispectral data. Then layers of two images were stacked to do next procedure of segmentation. An image stack is a step to compose four bands (RGB and NIR) with the spectral characteristics of the multispectral data and the spatial resolution of the panchromatic band (Vrabel,1996). There are different pansharpening algorithms in ENVI 5.1 (Excelis Visual Information Systems, Boulder, Colorado) and researchers found the GramSchmidt Sharpening algorithm (Labem and Brower, 2000) returned finer resolution pixels with consistent spectral characteristics. To improve the capability for segmenting small parcels, the Gram-Schmidt algorithm was applied in SPOT7 and returns pan-sharpened data with 4 bands. Then layers of two images were stacked to do the first step of object-based classification, image segmentation(Table 2), for RapidEye (t0) and pan- sharpened SPOT7 (t1) images are consistent in the blue, green, red and NIR band and their combination can be benefited to classification.

\subsection{Segmentation}

With the composed image (T), we segmented it into objects and used the multiresolution segmentation algorithm which, was in eCognition software. The multi-resolution segmentation algorithm uses a bottom-up region merging technique, with each pixel initialized as a single segment (Baatz \& Schäpe, 2000). If the degree of heterogeneity that is largely defined by the parameter scale, spatially adjacent objects will be merged. Once the defined scale parameter gets its value when there are no more possible, the process stops merges given. The greater the scale parameter, more heterogeneity allowed in each object, and the larger the average size of the objects. Two other parameters, color, and shape, can also be set to determine the relative weighting of reflectance and shape in defining segments. The total weighted value of color and shape equals to one (Trimble, 2012). Previous studies showed that a higher weight, typically up to 0.9 , which should be given to color for better segmentation results (Mathieu, Aryal, \& Chong, 2007; Pu, Landry, \& Yu, 2011). Therefore, we set the weights as 0.9 and 0.1 , respectively.

There are also two other values, compactness and smoothness which are set to determine spatially adjacent segments, the relative weighting of reflectance were determined by testing different parameter values and visually interpreting the image segmentation results. Therefore, we set the weights as $65,0.9$ and 0.1 , respectively. The image layer weights mean the weight coefficient of blue, green, red, NIR bands of each image $\left(t_{0}, t_{1}\right)$. And we also used NDVI and texture features in segmentation.

\begin{tabular}{|c|c|c|c|c|}
\hline Scale & $\begin{array}{c}\text { Image layer } \\
\text { weights }\end{array}$ & Shape & Compactness & Smothness \\
\hline 65 & $1: 1: 1: 1: 1: 1: 1: 1$ & 0.1 & 0.5 & 0.5 \\
\hline
\end{tabular}

Table 2. The parameters of segmentation

\subsection{Fuzzy classification}

After segmentation, features of shape, texture (e.g. Grey-level covariance matrix), vegetation index (e.g. NDVI) were used in the fuzzy classification of each image (t0, t1). When implementing the segmentation, we used it as the thematic layer to each image. Consequently, the generated objects were not allow to cross any of the borders separating different thematic classes of each classification map, and thus shared the boundaries of the different land cover class of the thematic layer. The use of a single segmentation for all the images is that it will not provide different objects because of change. In this step, we classified land use and cover types as vegetated and nonvegetated using spectral, and texture and a threshold of mean NDVI $>0$ or mean NDVI $\leqslant 0$ respectively. The aim of this step is to creat objects that were representative of the different ground features and prepare to select random samples for training and validating. 


\subsection{Sample selection}

For all the layers, a stratified random sampling method was used to obtain training samples. Samples were selected from within the two classification maps using expert knowledge of the study site for the following classes: bare land, arable land, built-up land, industrial and mining land, forest land and water area.

The training objects for each class were selected from multiple locations throughout the image, in order to obtain a representative sample. Furthermore, we used Google Earth to assist visual interpretation of high spatial resolution image data. We generated a total number of 600 sample points for classification of 2012 and 2015, with at least 40 samples for each class(Fuller, Smith et al. 2003). $70 \%$ of samples were used as training samples and rest of them were as testing samples.

\subsection{Classification in $\mathbf{R}$}

Training samples can be trained in $\mathrm{R}$ where we can use packages to improve the efficiency of classification, for it takes the shorter time than the same progress in eCognition when texture features are used in classification. The samples were exported from eCognition into $\mathrm{R}$ and samples in open source software are treated as statistical files. The final trained model was applied to all objects using the 'predict' function in R.

Map 2012 and 2015 was classified by the same classification method above. At last the land cover Map of 2012 and 2015 were classified as 7 land use/cover classes: bare land, arable land, built-up land, industrial and mining land, forest land and water area by using support vector machine (SVM) and objectbased method.

SVM is used to train a support vector machine. It can be used to carry out general regression and classification, as well as density estimation. A formula interface is provided in "e1071" package in R. SVMs are a class of kernel-based machine learning algorithms, which have been shown to be effective for classifying a wide variety of data types, including remotesensing data (Mountrakis, Im, and Ogole 2011; Richards and Kingsbury 2014). We implemented SVM classification using segmented two images were first linearly rescaled. Rescaling in this way has problems created by differences in the range of different attributes. For the kernel model, we selected the radial basis function (RBF), which is capable of mapping both nonlinear and linear of input values into higher-dimensional classification space (Keerthiand Lin 2003). Optimal values of the penalty $(\mathrm{C})$ and kernel $(\gamma)$ parameters for the RBF model were selected which in this study is 1300 and 0.0001 . Once optimal parameter values were determined, the SVM model was applied to all training samples in the images using the Training tool from $\mathrm{R}$, and then applied to all the objects, yielding a classified product.

\subsection{Change detection}

Multi-temporal/multi-date object change detection uses a single segmentation of all the stacked images. And image objects have same geometric properties at two times. What's more, it exploits the geometrical, spectral, and derived features to create change paths. Image segmentation and classification is applied directly to stacked multi-temporal image which produces spatially corresponding to change objects. The composite image used in Multi-temporal/multi-date object change detection may comprise one or more co-registered panchromatic, multispectral band, texture, or only spectral form multi-temporal images. But Multi-temporal/multi-date object change detection do not provide new/different objects that might be created at different times because of change(Hussain, Chen et al. 2013).

Desclée et al. (2006) stacked a time series of SPOT images and produced segmentation. Then the extracted objects were given the mean and standard deviation spectral values from each image of different dates. A procedure in GIS was used to identify changed objects as statistical tools. The unnatural values explained difference statistics referred to the changed objects. Change detection accuracy used error matrix $f$ and the accuracy was greater than $90 \%$ and an overall kappa was higher than 0.80. Bontemps et al. (2008) who applied segmentation to a series of time of spectral data. All the objects obtained spectral features, and used a Mahalanobis distance algorithm for change detection, that is one part of Multi-temporal/multi-date object change detection. Conchedda et al. (2008) and Stow et al. (2008) used multi-temporal stacked images in both segmentation and classification steps to get vegetation change objects and map them. There is also gathering on a series of date objects to analyze deforestation (Duveiller et al., 2008).

The single segmentation of all the stacked imaged results in creating image-objects consistent in size, shape, and location coordinate over time. Multi-temporal segmentation would also result in some artifacts if there are misregistration and differences in shadowing between dates (Stow, 2010). Another issue pertaining to the use of a single segmentation for all the images is that it will not provide new objects that might be created at different times because of change.

According to Multi-temporal/multi-date object change detection, and the GIS-based overlay of classification map of 2012 and 2015 , the change areas can be found and then masked. The high reflectance variability within individual features and the number of classes present in the high-spatial-resolution image can take change detection analysis in objects, not traditional pixel-based. The object-based image analysis (OBIA) techniques have been shown to reduce the effects of geo-referencing, higher spectral variability, and acquisition characteristics.

Multi-temporal/multi-date object change detection can extract change regions of all classes from 2012 to 2015 and we can obtain classification results of change analysis in GIS, for we used same segmentation from stacked images and objects of two classification maps correspond. Then Map 2015 and 2012 was masked to get change region layer. In Figure 3, land use and cover types decreased in Map 2012 corresponding to the types increased in Map 2015 and that present the changes in the study area. With change analysis, we can not only obtain land use/cover change map, but also the class hierarchy.

\subsection{Accuracy}

Classification method has to be evaluated by error matrix to specify the performances of the object-based method. Error matrices include the overall accuracies, user's and producer's accuracy, and the Kappa statistics. It can prove that classification method is really effective and suitable for the study area.

We conducted the accuracy assessment for 6 classes. These included the two classification maps, Map 2012 from Rapideye and Map 2015 from SPOT7 For all the maps, we used a stratified random sampling scheme to generate the checking points. For the classification maps, we used 30\% random samples selected in two images which have 180 objects of samples. We used reference data created from visual interpretation of very high spatial resolution image data. Error matrices were generated to calculate the overall accuracies, user's and producer's accuracy, and the Kappa statistics 

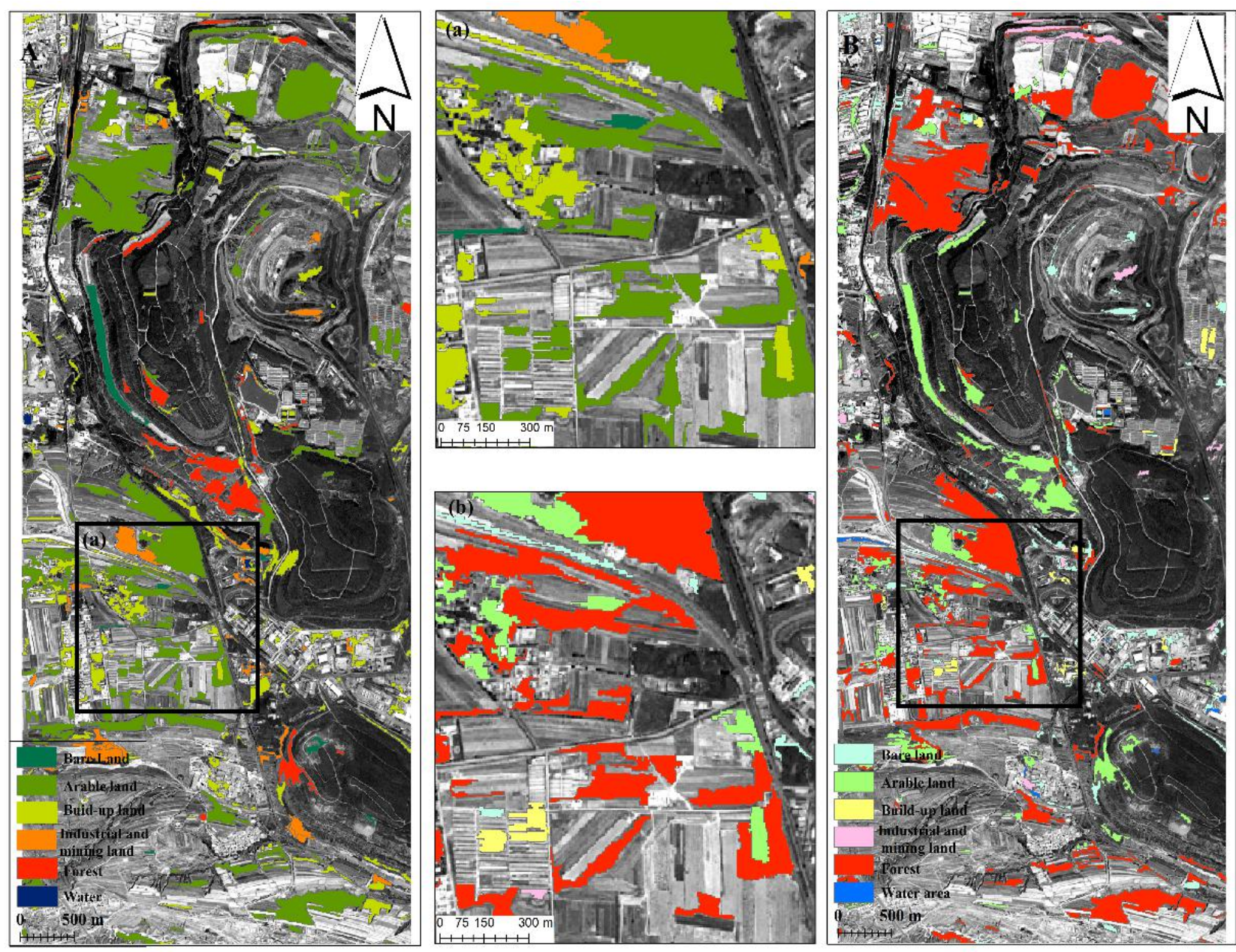

Figure 3. Panel A, the increased land cover class of Map 2015; panel B, the decreased land cover class of Map 2012

\section{RESULTS AND DISCUSSION}

Table 3 shows that the object-based classification results of mining area have high accuracies, and the overall accuracy and kappa coefficient of Map 2012 are $86.67 \%$ and $84.00 \%$, and of Map 2015 are $90.44 \%$ and $89.33 \%$ based on confusion matrixes. With object-based classification and change detection in the mining area, mine monitoring could be operated in local scale and changes were identified in short time interval.

\begin{tabular}{|l|l|l|l|l|l|l|l|l|}
\hline \multirow{2}{*}{ Land-use type } & \multicolumn{7}{|c|}{ Accuracies } & \multirow{2}{*}{ Overall Kappa statistics(p1/p2) } \\
\cline { 2 - 8 } & \multicolumn{2}{|l|}{ Producer's(p1/p2) } & \multicolumn{2}{|c|}{ User's(p1/p2) } & \multicolumn{2}{l|}{ Overall(p1/p2) } & - \\
\hline Bare land & 96.67 & 90.00 & 87.88 & 90.00 & - & - & - & - \\
Arable land & 93.33 & 85.00 & 77.78 & 95.00 & - & - & - & - \\
Build-up land & 83.33 & 90.00 & 100.00 & 80.61 & - & - & - & - \\
Mining land & 90.00 & 75.00 & 93.10 & 100.00 & - & - & - & - \\
Forest land & 83.33 & 83.00 & 83.33 & 90.00 & - & - & - & - \\
Water area & 90.00 & 75.00 & 100.00 & 86.67 & - & - & - & 89.33 \\
\hline
\end{tabular}

Table 3. Error matrices for both the land cover classification of Map 2012 (p1) and Map 2015 (p2) (\%)

\begin{tabular}{|l|l|l|l|l|l|l|l|}
\hline 2015 & Bare land & Arable land & Build-up land & Mining land & Forest land & Water area & Total 2015 \\
\hline Bare land & 0 & 115750 & 0 & 0 & 11325 & 0 & 127075 \\
Arable land & 21250 & 0 & 57300 & 18200 & 1509350 & 0 & 1606100 \\
Build-up land & 200300 & 344525 & 0 & 86050 & 681250 & 29075 & 1341200 \\
Mining land & 72475 & 114225 & 44450 & 0 & 52800 & 0 & 283950 \\
Forest land & 28750 & 258925 & 14775 & 36400 & 0 & 1975 & 340825 \\
Water area & 0 & 0 & 0 & 8750 & 0 & 0 & 8750 \\
Total 2012 & 322775 & 833425 & 116525 & 149400 & 2254725 & 31050 & 3707900 \\
\hline
\end{tabular}

Table 4. The pivottable of changed area from 2012 to $2015(\mathrm{~m} 2)$ 
As we can see, the environmental disturbance of mining land is always at a high level. Even more serious, mining completely destroys the native ecosystem and land reclamation needs to reconstruct a new one which must be adapted to local condition. In Table 4, land use and cover change is a dynamic process. Part of mining area was reclaimed to vegetation which is called pioneer plants to recover ecosystem and later would be used as arable land. The vegetation area changed from $46 \%$ to $40 \%$ accounted for the proportion of the total area from 2012 to 2015 , and the sum of arable land and vegetation area increased from $51 \%$ to $70 \%$. It represents that reclamation land has been finally used as normal land, and the local ecosystem has been recovered slowly. Although bare land in mining area has been decreased, it is obvious that some of arable land and forest land changed to bare land, the reclaimed land still needs to protect and find better ways to adapt to the local environment. There was still some kind of land converted to industrial and mine, but the trend of mining area is decreasing. Coal mining is still in production, but a great deal of them have been reclaimed. Pingshuo open-pit coal mine seems to work as usual, while to take pollution control and land reclamation. What's more, all types of land use and cover seems to change dynamically, for land use is also a dynamic process or there may be availability bias in classification, and we need to explore in the future study. It is of great importance that high monitoring accuracy in a short interval of real time for mining land, especially reclaimed mining land, so that we can get land use and cover information of short interval time in a local mining area.

Objects composed of similar pixels have details of shape, texture, spectral and spatial, while pixels just have the spectral feature to classify land use and cover types. Thus a pixel is not a true geographical object; rather, it is a representation of spectral values (digital numbers) in a grid whose boundaries lack real-world correspondence. Change detection based on object-based classification is similar to human understanding of geometry, who intuitively identifies the objects from an image rather than pixels by considering the different features (e.g. size texture, shape) and spatial arrangements(Addink, Van Coillie et al. 2012)

With the analytical framework in change detection becoming more data driven, it improves the capability of remote sensing data classification, because of a rapid increase in remote sensing data especially at high resolution, and increased computational power with more effective algorithms in open source software such as R, Python. With the development of object-based image analysis, the utility of machine learning is becoming more apparent, for it can help improve the classification results when objects are used by exploring different characteristics.

\section{CONCLUSION}

Although object-based classification and change detection have been successfully applied in urban sprawl, there are still few pieces of research of object-based classification and change detection about mining area including reclaimed land in a smaller scale and shorter time interval. In this study, we found that object-based classification and change detection in highresolution remote sensing image can not only be applied in urban change detection, but also to monitor land reclamation in mining land, and there is no doubt that it improves the monitoring accuracy.

As a result, object-based image analysis techniques were developed to handle the big changes in high-resolution data and to get better accuracy both in image classification and change detection, and with machine learning methods in $\mathrm{R}$ packages, the calculating ability and classification efficiency have been improved.

Multi-temporal/multi-date object change detection uses a single segmentation of all the stacked images in which image objects have same geometric properties at two times. Meanwhile, this change detection method uses the spatial, spectral, and derived features to classified objects and find changes. The advantages of using this method are to keep the geometry and the total number of objects in each image same, and easy to carry out change detection.

Object-based change detection delineates landscape features as real world objects and reduces the chance of misclassification. The machine learning can help improve the classification results when objects are used to explore different characteristics and understand the complex relationships. Object-based image analysis and change detection are now more frequently used, and they have great potential for answering the challenges of traditional change detection techniques on high-resolution images in the mining area.

The change detection of mining area can explain the local change in details and reflect the real situation in short time interval.

Nevertheless, we just used the object-based method without combined or compared to pixel-based approaches which are widely used in the future for time-series images. If pixel-based methods were taken consideration, it could meet more basic considerations and assumptions of these approaches.

We will test more machine learning methods in object-based classification and change detection, and then integrate or compared to pixel-based such as change vector analysis, principal component analysis, and vegetation index differencing. And more classes can be identified to take deeper analysis in the mining area in further analysis of the study.

\section{ACKNOWLEDGEMENTS}

This research was supported by National Science Foundation of China (NO. 41571508)

\section{REFERENCES}

Addink, E. A., F. M. B. Van Coillie and S. M. De Jong (2012). Introduction to the GEOBIA 2010 special issue: From pixels to geographic objects in remote sensing image analysis. International Journal of Applied Earth Observation and Geoinformation 15: 1-6.

Alkhudhairy, D. H. A., I. Caravaggi and S. Giada (2005). Structural Damage Assessments from Ikonos Data Using Change Detection, Object-Oriented Segmentation, and Classification Techniques. Photogrammetric Engineering \& Remote Sensing 71(7), 825-838.

BaatzGIT, M. and A. Schäpe (2000). An optimization approach for high quality multi-scale image segmentation. Beiträge zum A-Symposium.

Bai, Z. K., Xun, W. J. (2008). A case study on Pingshuo Mining Area: Land rehabilititation and reutilization in mining distericts." Resources \& Industries, 10(05), 32-37. (in Chinese)

Boerner, R. E. J., DeMers, M. N., Simpson, J. W., Artigas, F. J., Silva, A., \& Berns, L. A. (1996). Markov Models of Inertia and Dynamism on Two Contiguous Ohio Landscapes. Geographical Analysis, 28(1), 56-66. 
Bontemps, S., Bogaert, P., Titeux, N., Defourny, P., 2008. An object-based change detection method accounting for temporal dependences in time series with medium to coarse spatial resolution. Remote Sensing of Environment 112, 3181-3191.

Chen, G., G. J. Hay, L. M. Carvalho and M. A. Wulder (2012). Object-based change detection. International Journal of Remote Sensing 33(14), 4434-4457.

Chen, Z. Q. and T. C. Hutchinson (2007). Urban damage estimation using statistical processing of satellite images: 2003 Bam, Iran earthquake. Journal of Computing in Civil Engineering 21(3), 187-199.

Conchedda, G., Durieux, L., Mayaux, P., 2008. An objectbased method for mapping and change analysis in mangrove ecosystems. ISPRS Journal of Photogrammetry and Remote Sensing 63, 578-589

Demirel, N., M. K. Emil and H. S. Duzgun (2011). Surface coal mine area monitoring using multi-temporal high-resolution satellite imagery. International Journal of Coal Geology 86(1), $3-11$.

Durieux, L., Lagabrielle, E., Nelson, A., 2008. A method for monitoring building construction in urban sprawl areas using object-based analysis of Spot 5 images and existing GIS data. ISPRS Journal of Photogrammetry and Remote Sensing 63,399-408.

Duveiller, G., Defourny, P., Desclée, B., Mayaux, P., 2008. Deforestation in Central Africa: estimates at regional, national and landscape levels by advanced processing of systematicallydistributed Landsat extracts. Remote Sensing of Environment 112, 1969-1981.

Fuller, R. M., G. M. Smith and B. J. Devereux (2003). The characterisation and measurement of land cover change through remote sensing: problems in operational applications? International Journal of Applied Earth Observation \& Geoinformation 4(3), 243-253.

Hendrychová, M. and M. Kabrna (2016). An analysis of 200year-long changes in a landscape affected by large-scale surface coal mining: History, present and future. Applied Geography 74, 151-159.

Hussain, M., D. Chen, A. Cheng, H. Wei and D. Stanley (2013). Change detection from remotely sensed images: From pixelbased to object-based approaches. ISPRS Journal of Photogrammetry and Remote Sensing 80, 91-106.

Hu, Z. Q., Xie, H. Q. (2005). Study on land use/cover change of coal mining area based on remote sensing images. Journal of China Coal Society, 30(1), 44-48. (in Chinese)

Keerthi, S. S., and C.-J. Lin. 2003. Asymptotic Behaviors of Support Vector Machines with Gaussian Kernel. Neural Computation 15, 1667-1689.

Labem, C.A., and Brower, B.V., 2000. "Process for enhancing the spatial resolution of multispectral imagery using pansharpening." Technical Report US Patent \#6011875, Eastman, Kodak Company 2000.

Li, N., C. Yan and J. Xie (2015). Remote sensing monitoring recent rapid increase of coal mining activity of an important energy base in northern China, a case study of Mu Us Sandy Land. Resour. Conserv. Recycl 94, 129-135.
Lu, D., E. Moran, S. Hetrick and G. Li (2011). Land-use and land-cover change detection. Advances in Environmental Remote Sensing Sensors, Algorithms, and Applications. CRC Press Taylor \& Francis Group, New York, 273-290.

Mathieu, R., Aryal, J., \& Chong, A. K. (2007). Object-based classification of ikonos imagery for mapping large-scale vegetation communities in urban areas. Sensors, 7, 2860-2880.

Mountrakis, G., Im, J., \& Ogole, C. (2011). Support vector machines in remote sensing: a review. ISPRS Journal of Photogrammetry \& Remote Sensing, 66(3), 247-259.

Myint, S. W., P. Gober, A. Brazel, S. Grossman-Clarke and Q. Weng (2011). Per-pixel vs. object-based classification of urban land cover extraction using high spatial resolution imagery. Remote sensing of environment, 115(5), 1145-1161.

Pu, R. L., Landry, S., \& Yu, Q. (2011). Object-based urban detailed land cover classification with high spatial resolution IKONOS imagery. International Journal of Remote Sensing, 32, 3285-3308.

R Development Core Team (2011). R: A Language and Environment for Statistical Computing. $R$ Foundation for Statistical Computing, Vienna, Austria. ISBN 3-900051-07-0, http://www.R-project.org/.

Richards, J. A., \& Kingsbury, N. G. (2014). Is there a preferred classifier for operational thematic mapping? IEEE Transactions on Geoscience \& Remote Sensing, 52(5), 2715-2725.

Rodriguez-Galiano, V. F., B. Ghimire, J. Rogan, M. ChicaOlmo and J. P. Rigol-Sanchez (2012). An assessment of the effectiveness of a random forest classifier for land-cover classification. ISPRS Journal of Photogrammetry and Remote Sensing 67, 93-104.

Singh, A. (1989). Review Article Digital change detection techniques using remotely-sensed data. International Journal of Remote Sensing, 10(6), 989-1003.

Stow, D., Hamada, Y., Coulter, L., Anguelova, Z., 2008. Monitoring shrubland habitat changes through object-based change identification with airborne multispectral imagery. Remote Sensing of Environment, 112, 1051-1061.

Vrabel, J., 1996. Multispectral imagery band sharpening study. Photogramm. Eng. Remote Sens, 62 (9), 1075-1083.

Zhou, W., Cao, Y. G., Bai, Z. K., \& Wang, J. M. (2012). Indicators for Monitoring Land Reclamation in Coal Mining Area. China Land Sciences, 26(11), 68-73. (in Chinese) 